\title{
INVARIANT TORI FOR PERIODICALLY PERTURBED OSCILLATORS
}

\author{
Carmen Chicone
}

\begin{abstract}
The response of an oscillator to a small amplitude periodic excitation is discussed. In particular, sufficient conditions are formulated for the perturbed oscillator to have an invariant torus in the phase cylinder. When such an invariant torus exists, some perturbed solutions are in the basin of attraction of this torus and are thus entrained to the dynamical behavior of the perturbed system on the torus. In particular, the perturbed solutions in the basin of attraction of the invariant torus are entrained to a subharmonic or to a quasi periodic motion.
\end{abstract}

\section{Introduction}

Periodically perturbed nonlinear oscillators are widely studied for their rich dynamical behavior and because they are employed as mathematical models of many important physical phenomena. A fundamental problem of theoretical as well as practical interest is to determine the dynamics of a periodically perturbed oscillator near a resonance between a natural period of the oscillator and the period of the perturbation.

Here, we will consider the persistence of resonant invariant tori for periodically perturbed oscillators that have a one parameter family of periodic orbits such that the derivative of the corresponding period function with respect to the parameter has no critical points. The corresponding one parameter family of periodic orbits in the phase plane of such an oscillator is called a regular period annulus.

For a periodically perturbed oscillator with a regular period annulus, first order regular perturbation theory together with a reduction to the

This research was supported by the National Science Foundation under the grant DMS-9531811. 
Implicit Function Theorem can be used to show that there is a function defined on each resonant periodic orbit, the (subharmonic) "Melnikov" function, whose simple zeros correspond to families of initial points for perturbed periodic solutions, cf. [1], [2], [3], [6], [10]. However, it is possible - when the periodic perturbation contains only a few harmonicsthat only a few of the resonant orbits have associated Melnikov functions with simple zeros. In this case, the Melnikov functions associated with most of the remaining resonant orbits are of fixed sign, or they vanish identically. Thus, only a finite number of the resonances are excited at first order to produce perturbed periodic solutions. In fact, this is the case most often encountered in applied mathematics where, usually, only the "dominant" harmonics of a periodic perturbation are modeled.

An analysis using perturbation theory, carried to first order in the perturbation parameter, suggests that perturbed orbits, starting near a resonant unperturbed orbit with an associated Melnikov function of fixed sign, drift away from the vicinity of the resonant orbit in a direction determined by the sign, while perturbed orbits, starting near an unperturbed resonant orbit with an associated Melnikov function that vanishes, remain near the resonant orbit. If, in addition, the perturbation is dissipative, then this scenario indicates the presence of an invariant set for the perturbed flow near the resonant unperturbed orbits with vanishing Melnikov functions. This scenario is often valid. In fact, when this invariant set is an attractor, it often represents the dominant feature of the dynamics - most orbits will be in its basin of attraction. However, the proof of the existence of an invariant manifold, near a resonant unperturbed orbit with a vanishing Melnikov function, requires additional hypotheses that incorporate a rather delicate relationship among several quantities; especially, the rate of change of frequency with respect to the change in energy at the resonance, the strength of the damping, and the amplitude of the periodic perturbation. We will formulate the precise relationship among these quantities that ensures the existence of a Lipschitz continuous invariant torus. The conditions required for the existence of invariant tori with additional smoothness remains a problem for future research.

In Section 2 the main result on the existence of invariant tori will be formulated and proved. This result will be applied to a simple oscillator model in Section 3. A new construction of action angle variables will be given in Appendix A.

The author is very grateful to the referee and to Jorge Sotomayor for carefully reading a preliminary version of this paper. In particular, Sotomayor suggested an important improvement in the original formulation of the main result in Section 2. 


\section{An Existence Theorem for Invariant Tori}

\subsection{Resonance, Poincaré maps and Melnikov Functions.}

Consider a periodically perturbed planar oscillator given by

$$
\dot{u}=f(u)+\epsilon g(u, t)
$$

where the function $t \mapsto g(u, t)$ is $2 \pi / \Omega$ periodic, and where $\epsilon$ is a small parameter. Also, for each point $\zeta$ in the domain of definition of the system (1), let $t \mapsto u(t, \zeta, \epsilon)$ denote the solution of (1) with the initial condition $u(0, \zeta, \epsilon)=\zeta$.

We will assume that the unperturbed oscillator

$$
\dot{u}=f(u)
$$

has a regular period annulus $\mathcal{A}$. A periodic orbit $\Gamma$ in $\mathcal{A}$ with period $T$ is called resonant if there are relatively prime positive integers $m$ and $n$ such that

$$
m \frac{2 \pi}{\Omega}=n T
$$

If $\Gamma$ is a resonant orbit and $p \in \Gamma$, we define the associated (subharmonic) Melnikov function by

$$
M^{m: n}(\phi):=\int_{0}^{2 \pi m / \Omega} \mathcal{E}(t) f(u(t+\phi, p, 0)) \wedge g(u(t+\phi, p, 0), t) d t,
$$

where

$$
\mathcal{E}(t):=e^{-\int_{0}^{t} \operatorname{div} f(u(s+\phi, p, 0)) d s} .
$$

Clearly, the Melnikov function is T-periodic in $\phi$. Thus, it can be viewed as a function on $\Gamma$. Moreover, a different choice of base point $p \in \Gamma$ serves only to translate its graph.

If the unperturbed system is Hamiltonian, the usual case for applications, then the divergence of the unperturbed vector field vanishes, and, by a change of variables, the Melnikov function can be represented in the following convenient form

$$
M^{m: n}(\phi):=\int_{0}^{2 \pi m / \Omega} f(u(t, p, 0)) \wedge g(u(t, p, 0), t-\phi) d t .
$$

As is well known, the dynamics of (1) correspond to the dynamics of the (stroboscopic) Poincaré map $\zeta \mapsto P(\zeta, \epsilon)$ given by

$$
P(\zeta, \epsilon):=u(2 \pi / \Omega, \zeta, \epsilon) .
$$


For example, a periodic point of the Poincaré map lies on a periodic solution of (1), and an invariant simple closed curve for the Poincaré map corresponds to an invariant torus of (1) in the phase cylinder obtained by considering the time variable $t$ modulo $2 \pi / \Omega$. This provides a convenient geometric language that we will use to describe the dynamics of the oscillator. For example, a basic result of the subject is the following theorem $[\mathbf{1}]$.

Theorem 2.1. If the differential equation (1) has a regular period annulus $\mathcal{A}$ and if the Melnikov function $M^{m: n}$, defined on an $(m: n)$ resonant periodic orbit in $\mathcal{A}$, has a simple zero $\phi_{0}$, then there is a curve $\epsilon \mapsto \beta(\epsilon)$, defined in some open neighborhood of $\epsilon=0$, with $\beta(0)=u\left(\phi_{0}, p, 0\right)$ and $P^{m}(\beta(\epsilon), \epsilon) \equiv \beta(\epsilon)$. That is, $\beta(\epsilon)$ is the initial condition for a periodic orbit of the corresponding perturbed system.

\subsection{Action Angle Variables and Partial Averaging at a Res-} onance.

To study the dynamics of (1) near a periodic orbit $\Gamma$ contained in a period annulus $\mathcal{A}$, we will transform the system to angular standard form using a special "periodic" coordinate system defined in a neighborhood of $\Gamma$. This special coordinate system reduces to the action angle coordinate system in case the unperturbed oscillator (2) is Hamiltonian.

The precise result that we need is formulated in the following theorem. A proof of the theorem based on the integration of the planar variational equations is given in Appendix A.

Theorem 2.2. Suppose that $\Gamma$ is a periodic orbit of the differential equation (2). If $\Gamma$ is contained in a period annulus $\mathcal{A}$ of (2), then there is a smooth change of coordinates defined in an open subset of $\mathcal{A}$ that contains $\Gamma$ such that the differential equation (1), in the new coordinates $(I, \vartheta)$, has the form

$$
\dot{I}=\epsilon F(I, \vartheta, t), \quad \dot{\vartheta}=\omega(I)+\epsilon G(I, \vartheta, t),
$$

where both $F$ and $G$ are $2 \pi$ periodic in $\vartheta$ and $2 \pi / \Omega$ periodic in $t$.

For system (1) transformed into the new coordinates defined in Theorem 2.2 , we let $I_{0}$ denote the "action" such that the unperturbed $(m: n)$ resonant periodic orbit $\Gamma$ is given by the graph of $I=I_{0}$. Also, we note that the resonance relation (3), with respect to system (5), is given by

$$
m \frac{2 \pi}{\Omega}=n \frac{2 \pi}{\omega\left(I_{0}\right)}
$$


We will use the method of averaging to obtain a "normal form" for the system (5) at $\Gamma$; cf. [6], [8], [9] and, especially, [10, p. 143]. However, to apply the averaging procedure, we require a preliminary transformation of system (5) into the "time periodic standard form". The appropriate transformation is a well studied time dependent change of coordinates that localizes system (5) at the resonant orbit $\Gamma$. In fact, the transformation is defined, for $\epsilon \geq 0$, by

$$
I=I_{0}+\sqrt{\epsilon} \ell, \quad \vartheta=\omega\left(I_{0}\right) t+\sigma .
$$

If, after changing to these new coordinates in system (5), we Taylor expand to third order in powers of $\sqrt{\epsilon}$, and, if we use subscripts to denote partial derivatives, then the transformed system is given by

$$
\begin{aligned}
\dot{\ell}= & \sqrt{\epsilon} F\left(I_{0}, \omega\left(I_{0}\right) t+\sigma, t\right)+\epsilon F_{I}\left(I_{0}, \omega\left(I_{0}\right) t+\sigma, t\right) \ell \\
& +\epsilon^{3 / 2} F_{I I}\left(I_{0}, \omega\left(I_{0}\right) t+\sigma, t\right) \ell^{2}+O\left(\epsilon^{2}\right) \\
\dot{\sigma}= & \sqrt{\epsilon} \omega^{\prime}\left(I_{0}\right) \ell+\epsilon\left(G\left(I_{0}, \omega\left(I_{0}\right) t+\sigma, t\right)+\frac{1}{2} \omega^{\prime \prime}\left(I_{0}\right) \ell^{2}\right) \\
& +\epsilon^{3 / 2}\left(G_{I}\left(I_{0}, \omega\left(I_{0}\right) t+\sigma, t\right)+\frac{1}{6} \omega^{\prime \prime \prime}\left(I_{0}\right) \ell^{3}\right)+O\left(\epsilon^{2}\right),
\end{aligned}
$$

where the order symbol is used for notational convenience. This new system is in the correct form for averaging. In particular, the associated vector field is $2 \pi m / \Omega$ periodic in time.

We will require the averages and the properties of the functions appearing in (7). For example, we define

$$
\langle F\rangle(\sigma):=\frac{\Omega}{2 \pi m} \int_{0}^{2 \pi m / \Omega} F\left(I_{0}, \omega\left(I_{0}\right) t+\sigma, t\right) d t,
$$

and note that $\langle F\rangle$ is a $2 \pi$ periodic function. In fact, it should be clear that $\langle F\rangle$ is the subharmonic Melnikov function represented in the actionangle coordinates. The functions $\langle G\rangle(\sigma),\left\langle G_{I}\right\rangle(\sigma),\left\langle F_{I}\right\rangle(\sigma)$, and $\left\langle F_{I I}\right\rangle(\sigma)$ are defined similarly. Also, we will require several auxiliary functions:

$$
\begin{aligned}
& u_{1}(\sigma, t):=\int_{0}^{t} F\left(I_{0}, \omega\left(I_{0}\right) s+\sigma, s\right)-\frac{2 \pi m}{\Omega}\langle F\rangle(\sigma) d s \\
& v_{2}(\sigma, t):=\int_{0}^{t} G\left(I_{0}, \omega\left(I_{0}\right) s+\sigma, s\right)-\frac{2 \pi m}{\Omega}\langle G\rangle(\sigma)-\omega^{\prime}\left(I_{0}\right) u_{1}(\sigma, s) d s \\
& h(\sigma):=\frac{\Omega}{2 \pi m} \int_{0}^{2 \pi m / \Omega} F_{\vartheta}\left(I_{0}, \omega\left(I_{0}\right) t+\sigma, t\right) v_{2}(\sigma, t) \\
&+F_{I}\left(I_{0}, \omega\left(I_{0}\right) t+\sigma, t\right) u_{1}(\sigma, t) d t .
\end{aligned}
$$


The next theorem specifies the form of the third order averaged system corresponding to (7) as well as the form of the third order averaging transformation at a resonance where both $\langle F\rangle$ and $\langle G\rangle$ vanish. Our main result, to follow, states conditions for the existence of an invariant torus in terms of the quantities that appear in the third order averaged system. The next theorem is somewhat technical. However, it states that an averaging transformation exists that changes system (7) into the form that we will use to prove the persistence of the resonant torus corresponding to $\Gamma$.

Theorem 2.3. Consider the differential equation (7) and the functions defined as in (8). If $\langle F\rangle(\sigma) \equiv 0$ and $\langle G\rangle(\sigma) \equiv 0$, then there is an averaging transformation of the form

$$
\begin{aligned}
\ell & =\bar{\ell}+\sqrt{\epsilon} u_{1}(\bar{\sigma}, t)+\epsilon \bar{\ell} u_{2}(\bar{\sigma}, t)+\epsilon^{3 / 2} u_{3}(\bar{\ell}, \bar{\sigma}, t) \\
\sigma & =\bar{\sigma}+\epsilon v_{2}(\bar{\sigma}, t)+\epsilon^{3 / 2} v_{3}(\bar{\ell}, \bar{\sigma}, t)
\end{aligned}
$$

such that the transformation is $2 \pi m / \Omega$ periodic in $t$ and $2 \pi$ periodic in $\bar{\sigma}$, the functions $u_{1}$ and $v_{2}$ are as defined in (9), and such that the system (7) in the new coordinates has the form

$$
\begin{aligned}
\dot{\bar{\ell}}= & \epsilon\left\langle F_{I}\right\rangle(\bar{\sigma}) \bar{\ell}+\epsilon^{3 / 2}\left(\left\langle F_{I I}\right\rangle(\bar{\sigma}) \bar{\ell}^{2}+h(\bar{\sigma})\right)+\epsilon^{2} K(\bar{\ell}, \bar{\sigma}, t, \epsilon), \\
\dot{\bar{\sigma}}= & \sqrt{\epsilon} \omega^{\prime}\left(I_{0}\right) \bar{\ell}+\epsilon \frac{1}{2} \omega^{\prime \prime}\left(I_{0}\right) \bar{\ell}^{2} \\
& +\epsilon^{3 / 2}\left(\left\langle G_{I}\right\rangle(\bar{\sigma})+\frac{1}{6} \omega^{\prime \prime \prime}\left(I_{0}\right) \bar{\ell}^{3}\right)+\epsilon^{2} L(\bar{\ell}, \bar{\sigma}, t, \epsilon),
\end{aligned}
$$

where the function $h$ is defined as in (9), and both of the functions $K$ and $L$ are $2 \pi m / \Omega$ periodic in $t$ and $2 \pi$ periodic in $\bar{\sigma}$.

Proof: To prove the theorem, we apply the usual algorithm for obtaining the third order averaged system while checking that the averaging transformation can be taken to be as in (10). The steps are straightforward: Differentiate the transformation (10) with respect to $t$ and substitute the target system (11) into the result retaining terms to order $\epsilon^{3 / 2}$. Next, substitute the transformation (10) into (7) and Taylor expand the result, as a function of $\epsilon$, to order $\epsilon^{3 / 2}$. Equating the coefficients of equal powers of $\epsilon$ in the two systems just defined, we obtain equations for the time derivatives of the coefficients of the order parameter in the averaging transformation. Taken in turn with respect to the powers of the order parameter, all of these equations have the form $w_{t}=\rho(t)$ where $\rho$ 
is a function with zero average and $w$ is one of the unknown coefficients. Thus, if each of these equations for the coefficients is solved in the form

$$
w=\int_{0}^{t} \rho(s) d s,
$$

then the coefficients have the required periodicity properties.

\subsection{The Main Result.}

For notational convenience, we will consider an abstract differential equation that is a generalization of (11); namely,

$$
\begin{aligned}
& \dot{\ell}=\mu^{2} p(\sigma) \ell+\mu^{3}\left(q(\sigma) \ell^{2}+r(\sigma)\right)+\mu^{4} R(\ell, \sigma, t, \mu), \\
& \dot{\sigma}=\mu \lambda \ell+\mu^{2} \nu \ell^{2}+\mu^{3} S(\ell, \sigma, t, \mu),
\end{aligned}
$$

where $p, q$, and $r$ are $2 \pi$ periodic functions, $\lambda, \mu$, and $\nu$ are real numbers, and both of the functions $R$ and $S$ are $2 \pi m / \Omega$ periodic in $t$ and $2 \pi$ periodic in $\sigma$. We will study the existence of invariant tori for (12) when $\mu$ is considered to be a small parameter.

As an instructive example, consider the special case of (12) given by

$$
\dot{\ell}=-\mu^{2} M \ell+\mu^{3} A \sin \sigma, \quad \dot{\sigma}=\mu \lambda \ell+\mu^{2} \nu \ell^{2},
$$

where $M>0, \lambda>0, \mu>0$, and $A$ are constants, and $(\ell, \sigma)$ are considered as coordinates on a cylinder; that is, $\sigma$ is an angular coordinate modulo $2 \pi$.

The points with coordinates $(0,0)$ and $(0, \pi)$ are rest points of the differential equation (13). The rest point at $(0,0)$ is a hyperbolic saddle, while the rest point at $(0, \pi)$ is a hyperbolic sink. Moreover, the eigenvalues at the sink are

$$
\frac{\mu^{2}}{2}\left(-M \pm \sqrt{M^{2}-4 \lambda A}\right) .
$$

If $M^{2}-4 \lambda A<0$, then the sink is of spiral type, while if $M^{2}-4 \lambda A>0$, the sink is nodal.

Clearly, the invariant set formed by the union of the sink and the unstable manifold of the saddle is a manifold only in the case $M^{2}-4 \lambda A \geq 0$. If, on the other hand, $M^{2}-4 \lambda A<0$, the normal contraction is not strong enough to prevent the "roll up" of the invariant set at the sink, thus destroying the differentiability of the invariant set at that point, cf. [7]. Hence, we can not expect to have an invariant 
manifold for the system (12) unless there is an appropriate relationship among the quantities in (12) corresponding to the variables in the example given by the normal contraction rate, $M$; the twist rate on the unperturbed invariant set, $\lambda$; and the amplitude of the periodic perturbation, $A$.

A sufficient condition for the existence of a Lipschitz continuous invariant torus for system (12) will be formulated in Theorem 2.4 below. In order to state the theorem, let $\mathcal{C}$ denote the set of continuous functions mapping $\mathbb{R} \times \mathbb{R} \rightarrow \mathbb{R}$ that are $2 \pi$ periodic in their first argument and $2 \pi m / \Omega$ periodic in their second argument. We define two Banach spaces: $\mathcal{C}^{0}$ and $\mathcal{C}^{0,1}$. The Banach space $\mathcal{C}^{0}$ consists of all elements of $\mathcal{C}$ bounded with respect to the usual uniform norm given by

$$
\|H\|=\sup _{(s, \tau)}|H(s, \tau)|,
$$

while the Banach space $\mathcal{C}^{0,1}$ consists of all elements of $\mathcal{C}$ bounded with respect to the norm given by

$$
\|H\|_{0,1}=\|H\|+\sup _{(s, \tau) \neq(\bar{s}, \bar{\tau})} \frac{|H(s, \tau)-H(\bar{s}, \bar{\tau})|}{|s-\bar{s}|+|\tau-\bar{\tau}|} .
$$

Also, we note that a $2 \pi$ periodic function or a $2 \pi m / \Omega$ periodic function of a single variable may be regarded as an element of $\mathcal{C}$. Thus, the uniform norm and the $(0,1)$ norm are defined for such functions as well. Moreover, the second summand in the definition of the $(0,1)$ norm is also called the Lipschitz constant of $H$ and is denoted $\operatorname{Lip}(H)$. The Lipschitz constant can be defined in the same manner for functions of several variables.

Theorem 2.4. Consider the differential equation (12) and define

$$
M:=\min _{0 \leq \sigma \leq 2 \pi}|p(\sigma)|>0
$$

If $\lambda \neq 0$,

$$
\begin{aligned}
& 5 M>\operatorname{Lip}(p), \\
& M^{2} \geq 6|\lambda|\|r\|_{0,1},
\end{aligned}
$$

and if $|\mu|$ is sufficiently small, then there is a function $H \in \mathcal{C}^{0,1}$ such that the set $\{(\ell, \sigma, t): \ell=H(\sigma, t)\}$ is an invariant torus for (12).

Proof: We will prove the theorem under the assumption that $p$ is a positive function. The case when $p$ is negative follows by simply reversing the direction of time. 
For each $H \in \mathcal{C}^{0,1}$ with $\|H\|_{0,1} \leq 1$ and for each number $\mu$ with $|\mu| \leq 1$, define a new differential equation by

$$
\begin{aligned}
& \dot{\ell}=\mu^{2} p(\sigma) \ell+\mu^{3}\left(q(\sigma) H^{2}(\sigma, t)+r(\sigma)\right)+\mu^{4} R(H(\sigma, t), \sigma, t, \mu), \\
& \dot{\sigma}=\mu \lambda H(\sigma, t)+\mu^{2} \nu H^{2}(\sigma, t)+\mu^{3} S(H(\sigma, t), \sigma, t, \mu) .
\end{aligned}
$$

The differential equation (12) is defined on an annular domain containing the closed curve on the cylinder given by $\ell=0$. In order to ensure that solutions and Lipschitz constants remain bounded, we choose some $\ell_{0}>0$ so that $(\ell, \sigma)$ is in the domain of definition of the differential equation as long as $|\ell|<\ell_{0}$, and we fix a smooth "bump function" $\psi: \mathbb{R} \rightarrow[0,1]$ such that $\psi(\ell) \equiv 1$ for $|\ell|<\ell_{0} / 2$ while $\psi(\ell) \equiv 0$ for $|\ell| \geq \ell_{0}$. Using the function $\psi$, we define new functions

$$
\begin{aligned}
\mathcal{R}(\sigma, t, H, \mu) & :=\psi(H(\sigma, t)) R(H(\sigma, t), \sigma, t, \mu), \\
\mathcal{S}(\sigma, t, H, \mu) & :=\psi(H(\sigma, t)) S(H(\sigma, t), \sigma, t, \mu), \\
\mathcal{Q}(\sigma, t, H, \mu) & :=q(\sigma) H^{2}(\sigma, t)+r(\sigma)+\mu \mathcal{R}(\sigma, t, H, \mu),
\end{aligned}
$$

and the following differential equation

$$
\begin{aligned}
& \dot{\ell}=\mu^{2} p(\sigma) \ell+\mu^{3} \mathcal{Q}(\sigma, t, H, \mu), \\
& \dot{\sigma}=\mu \lambda H(\sigma, t)+\mu^{2} \nu H^{2}(\sigma, t)+\mu^{3} \mathcal{S}(\sigma, t, H, \mu),
\end{aligned}
$$

that is equivalent to system (16) as long as $|\ell|<\ell_{0} / 2$.

Note that the second equation of the system (17) is decoupled from the system. We let $t \mapsto \sigma(t, s, \tau, H, \mu)$ denote its solution with the initial condition $\sigma(\tau, s, \tau, H, \mu)=s$. In view of the periodicity of the differential equation and the nature of the "cut off" function $\psi$, all such solutions are complete.

Choose $H \in \mathcal{C}^{0,1}$ and consider the associated differential equation (17). For $G \in \mathcal{C}^{0,1}$, the set $\{(\ell, \sigma, t): \ell=G(\sigma, t)\}$ is an invariant torus for this differential equation if and only if

$$
\begin{array}{r}
\frac{d}{d t} G(\sigma(t, s, \tau, H, \mu), t)=\mu^{2} p(\sigma(t, s, \tau, H, \mu)) G(\sigma(t, s, \tau, H, \mu), t) \\
+\mu^{3} \mathcal{Q}(\sigma(t, s, \tau, H, \mu), t, H, \mu) .
\end{array}
$$

Define

$$
\Phi(t, \tau, H):=e^{-\mu^{2} \int_{\tau}^{t} p(\sigma(u, s, \tau, H, \mu)) d u},
$$

and note that, if $t \geq \tau$, then

$$
|\Phi(t, \tau, H)| \leq e^{-\mu^{2} M(t-\tau)} .
$$


Using variation of parameters relative to the fundamental solution $\Phi$ of the differential equation obtained from the equation (18) by setting $\mathcal{Q}=0$, and the obvious change of variables, we find that

$$
\begin{aligned}
& \Phi(t, \tau, H) G(\sigma(t, s, \tau, H, \mu), t)-G(s, \tau) \\
& \quad=\int_{0}^{t-\tau} \mu^{3} \Phi(v+\tau, \tau, H) \mathcal{Q}(\sigma(v+\tau, s, \tau, H, \mu), v+\tau, H, \mu) d v .
\end{aligned}
$$

Under the assumption that $|\mu|>0$ and in view of the inequality (19), if we pass to the limit $t \rightarrow \infty$ in equation (20), then we obtain the equation

$$
G(s, \tau)=-\int_{0}^{\infty} \mu^{3} \Phi(t+\tau, \tau, H) \mathcal{Q}(\sigma(t+\tau, s, \tau, H, \mu), t+\tau, H, \mu) d t
$$

In other words, we have $G=\Lambda(H)$, where $\Lambda$ is the operator defined on the unit ball of $\mathcal{C}^{0,1}$ by

$$
\begin{aligned}
& \Lambda(H)(s, \tau) \\
& \quad=-\int_{0}^{\infty} \mu^{3} \Phi(t+\tau, \tau, H) \mathcal{Q}(\sigma(t+\tau, s, \tau, H, \mu), t+\tau, H, \mu) d t
\end{aligned}
$$

A fixed point of $\Lambda$ corresponds to the desired invariant torus. We have just shown that an invariant manifold given as the graph of an element of $\mathcal{C}^{0,1}$ is a fixed point of $\Lambda$. It is easy to show that, conversely, the graph of a fixed point of the operator $\Lambda$ is a Lipschitz continuous invariant torus.

We will use the Contraction Principle to show that $\Lambda$ has a fixed point. For this, we will construct a ball

$$
B_{\delta}:=\left\{H \in \mathcal{C}^{0,1}:\|H\|_{0,1} \leq \delta\right\}
$$

such that $\Lambda: B_{\delta} \rightarrow B_{\delta}$ and such that $\Lambda$ is a contraction on the set $B_{\delta}$ with respect to the uniform norm. The Contraction Principle is applicable because $B_{\delta}$ is a closed subset of $\mathcal{C}^{0}$, and, therefore, a complete metric space in the uniform norm.

To prove that the operator $\Lambda$ is a contraction on $B_{\delta}$ requires a series of estimates.

We note that both of the functions $\mathcal{R}$ and $\mathcal{S}$, appearing in the differential equation (17), are Lipschitz continuous with respect to all of their arguments. Under our assumptions that $\|H\|_{0,1} \leq \delta \leq 1$ and $0<|\mu| \leq 1$, we have

$$
\|\mathcal{R}\|<\infty, \quad\|\mathcal{S}\|<\infty
$$


as well as the fact that there are positive constants $\operatorname{Lip}(\mathcal{R})$ and $\operatorname{Lip}(\mathcal{S})$ such that

$$
\begin{aligned}
|\mathcal{R}(\sigma, t, H, \mu)-\mathcal{R}(\bar{\sigma}, \bar{t}, H, \mu)| & \leq \operatorname{Lip}(\mathcal{R})(|\sigma-\bar{\sigma}|+|t-\bar{t}|), \\
|\mathcal{S}(\sigma, t, H, \mu)-\mathcal{S}(\bar{\sigma}, \bar{t}, H, \mu)| & \leq \operatorname{Lip}(\mathcal{S})(|\sigma-\bar{\sigma}|+|t-\bar{t}|) .
\end{aligned}
$$

In addition, using the obvious estimates, there are uniform and Lipschitz estimates for $\mathcal{Q}$. In fact, the estimates that we will use are given by

$$
\begin{aligned}
& |\mathcal{Q}(\sigma, t, H, \mu)-\mathcal{Q}(\bar{\sigma}, \bar{t}, H, \mu)| \\
& \quad \leq \| r^{\prime}|||\sigma-\bar{\sigma}|+\left(2\|q\|_{0,1} \delta^{2}+|\mu| \operatorname{Lip}(\mathcal{R})\right)(|\sigma-\bar{\sigma}|+|t-\bar{t}|),
\end{aligned}
$$

$$
\begin{aligned}
& |\mathcal{Q}(\sigma, t, H, \mu)-\mathcal{Q}(\bar{\sigma}, t, \bar{H}, \mu)| \leq\left(\left\|r^{\prime}\right\|+|\mu| \operatorname{Lip}(\mathcal{R})\right. \\
& \left.\quad+\delta^{2}\|q\|_{0,1}\right)|\sigma-\bar{\sigma}|+(2 \delta\|q\|+|\mu| \operatorname{Lip}(\mathcal{R}))\|H-\bar{H}\| .
\end{aligned}
$$

We need estimates for the sizes of $\mu$ and $\delta$ that will ensure that the operator $\Lambda$ has image in $B_{\delta}$. For this, we must estimate the size of the $(0,1)$ norm of each element in the range of $\Lambda$. This will be done in two steps: we will estimate the uniform norm of an element in the range of $\Lambda$, and then we will obtain a Lipschitz estimate.

Using the definition of $\Lambda$ given in (21), the inequalities (19) and (22), and an integration, we obtain the following estimate for the uniform norm:

$$
\|\Lambda(H)\| \leq \frac{|\mu|}{M}\left(\|q\| \delta^{2}+\|r\|+|\mu|\|R\|\right) .
$$

To determine a Lipschitz estimate, we begin by using the inequality (23) to obtain the inequality

$$
\begin{array}{r}
|\Lambda(H)(s, \tau)-\Lambda(H)(\bar{s}, \bar{\tau})| \leq|\mu|^{3} \int_{0}^{\infty} e^{-\mu^{2} M t}\left\{\left\|r^{\prime}\right\|\left|\sigma_{1}(t)-\sigma_{2}(t)\right|\right. \\
\left.+\left(2\|q\|_{0,1} \delta^{2}+|\mu| \operatorname{Lip}(\mathcal{R})\right)\left(\left|\sigma_{1}(t)-\sigma_{2}(t)\right|+|\tau-\bar{\tau}|\right)\right\} d t
\end{array}
$$

where

$$
\sigma_{1}(t):=\sigma(t+\tau, s, \tau, H, \mu), \quad \sigma_{2}(t):=\sigma(t+\bar{\tau}, \bar{s}, \bar{\tau}, H, \mu) .
$$


Note that both $t \mapsto \sigma_{1}(t)$ and $t \mapsto \sigma_{2}(t)$ are solutions of the differential equation

$$
\dot{\sigma}=\mathcal{G}(\sigma, t, H, \mu):=\mu \lambda H(\sigma, t)+\mu^{2} \nu H^{2}(\sigma, t)+\mu^{3} \mathcal{S}(\sigma, t, H, \mu),
$$

and, as a result, we have

$$
\begin{aligned}
\mid \sigma_{1}(t)- & \sigma_{2}(t)|\leq| s-\bar{s} \mid \\
& +\int_{0}^{t}\left|\mathcal{G}\left(\sigma_{1}(v), v+\tau, H, \mu\right)-\mathcal{G}\left(\sigma_{2}(v), v+\bar{\tau}, H, \mu\right)\right| d v .
\end{aligned}
$$

We will use the following easily obtained Lipschitz estimate for $\mathcal{G}$ :

$$
|\mathcal{G}(\sigma, t, H, \mu)-\mathcal{G}(\bar{\sigma}, \bar{t}, H, \mu)| \leq \mu^{2} \gamma(\delta, \mu)(|\sigma-\bar{\sigma}|+|t-\bar{t}|),
$$

where

$$
\gamma(\delta, \mu):=|\lambda| \frac{\delta}{|\mu|}+2|\mu||\nu| \delta^{2}+|\mu| \operatorname{Lip}(\mathcal{S}) .
$$

When the estimate (29) is inserted into the inequality (28), we find that

$$
\begin{aligned}
& \left|\sigma_{1}(t)-\sigma_{2}(t)\right| \leq|\mu|^{2} \gamma(\delta, \mu)|\tau-\bar{\tau}| t \\
& \quad+|\mu|^{2} \gamma(\delta, \mu) \int_{0}^{t}\left|\sigma_{1}(v)-\sigma_{2}(v)\right| d v+|s-\bar{s}| .
\end{aligned}
$$

To obtain an estimate for the quantity $\left|\sigma_{1}(t)-\sigma_{2}(t)\right|$ that appears in the inequality (30), we will use the "Specific" Gronwall Lemma [9]:

Lemma 2.5 (Gronwall's Lemma). Suppose that $\delta_{1}>0, \delta_{2} \geq 0$ and, $\delta_{3} \geq 0$ are constants and that $\phi$ is a continuous nonnegative function. If, for $t_{0} \leq t \leq t_{0}+T$, we have

$$
\phi(t) \leq \delta_{2}\left(t-t_{0}\right)+\delta_{1} \int_{t_{0}}^{t} \phi(s) d s+\delta_{3},
$$

then

$$
\phi(t) \leq\left(\frac{\delta_{2}}{\delta_{1}}+\delta_{3}\right) e^{\delta_{1}\left(t-t_{0}\right)}-\frac{\delta_{2}}{\delta_{1}}
$$

for $t_{0} \leq t \leq t_{0}+T$.

Indeed, an application of Gronwall's Lemma to the inequality (30) yields

$$
\left|\sigma_{1}(t)-\sigma_{2}(t)\right| \leq(|s-\bar{s}|+|\tau-\bar{\tau}|) e^{\mu^{2} \gamma(\delta, \mu) t}-|\tau-\bar{\tau}|
$$


We will also use the estimate

$$
\left|\sigma_{1}(t)-\sigma_{2}(t)\right| \leq(|s-\bar{s}|+|\tau-\bar{\tau}|) e^{\mu^{2} \gamma(\delta, \mu) t}
$$

To obtain convergence of the improper integral in inequality (26), we must restrict the size of $\gamma$. For this, let $k=2-\sqrt{2}<1$ denote the critical point of the function

$$
x \mapsto \frac{1}{x}\left(1+\frac{1}{1-x}\right), \quad 0<x<1,
$$

and note that the corresponding critical value is given by

$$
\kappa:=\frac{1}{k}\left(1+\frac{1}{1-k}\right)=3+2 \sqrt{2}<6 .
$$

Also, define

$$
\delta=k \frac{M}{|\lambda|}|\mu|
$$

and note that, with this definition, we have

$$
\gamma(\delta, \mu)=k M+O(|\mu|)
$$

In particular, for $|\mu|$ sufficiently small, we have $\gamma(\delta, \mu)<M$.

Returning to the estimate (26), we insert the inequality (32) for the first occurrence of the quantity $\left|\sigma_{1}(t)-\sigma_{2}(t)\right|$, we insert the inequality (31) for its second occurrence, and then we integrate the resulting expression. After using the definition (34), we obtain the following estimate

(36) $|\Lambda(H)(s, \tau)-\Lambda(H)(\bar{s}, \bar{\tau})|$

$$
\leq|\mu|\left(\frac{\left\|r^{\prime}\right\|}{M-\gamma(\delta, \mu)}+O(|\mu|)\right)(|s-\bar{s}|+|\tau-\bar{\tau}|) .
$$

Using the inequalities (25) and (36), and the definition (34), it follows easily that

$$
\|\Lambda(H)\|_{0,1} \leq|\mu|\|r\|_{0,1}\left(\frac{1}{M}+\frac{1}{M-\gamma(\delta, \mu)}\right)+O\left(|\mu|^{2}\right)
$$


In addition, using the inequality (35), the definition of $\kappa$ from (33), and the formula for $\delta$ given in equation (34), we have

$$
\begin{aligned}
\|\Lambda(H)\|_{0,1} & \leq \frac{|\mu|\|r\|_{0,1}}{M}\left(1+\frac{1}{1-k}\right)+O\left(|\mu|^{2}\right) \\
& \leq \frac{|\mu|\|r\|_{0,1}}{M} k \kappa+O\left(|\mu|^{2}\right) \\
& \leq \frac{|\lambda|\|r\|_{0,1}}{M^{2}} \kappa \delta+O\left(|\mu|^{2}\right) .
\end{aligned}
$$

By rearranging the inequality (15), we have

$$
\frac{\|r\|_{0,1}|\lambda|}{M^{2}} \leq \frac{1}{6}
$$

Hence, in view of the fact that $\kappa<6$, if we take $|\mu|$ sufficiently small in the inequality (38), we have

$$
\|\Lambda(H)\|_{0,1} \leq \delta .
$$

It remains to show that $\Lambda$ is a contraction. To do this, we must estimate

$$
|\Lambda(H)(s, \tau)-\Lambda(\bar{H})(s, \tau)|
$$

For notational convenience, we define

$$
\sigma_{3}(t):=\sigma(t+\tau, s, \tau, H, \mu), \quad \sigma_{4}(t):=\sigma(t+\tau, s, \tau, \bar{H}, \mu) .
$$

By adding and subtracting the term $\Phi(t+\tau, \tau, H) \mathcal{Q}\left(\sigma_{4}(t), t+\tau, H, \mu\right)$ under the integral obtained in the expression for (41), by applying the Triangle Law, and by using the estimate (19), we have that

$$
\begin{aligned}
& |\Lambda(H)(s, \tau)-\Lambda(\bar{H})(s, \tau)| \\
\leq & \int_{0}^{\infty}|\mu|^{3} e^{-\mu^{2} M t}\left|\mathcal{Q}\left(\sigma_{3}(t), t+\tau, H, \mu\right)-\mathcal{Q}\left(\sigma_{4}(t), t+\tau, \bar{H}, \mu\right)\right| d t \\
+ & \int_{0}^{\infty}|\mu|^{3}\left|\mathcal{Q}\left(\sigma_{4}(t), t+\tau, H, \mu\right)\right||\Phi(t+\tau, \tau, H)-\Phi(t+\tau, \tau, \bar{H})| d t .
\end{aligned}
$$

To estimate the first summand of the right hand side of the inequality (42), we apply the Lipschitz inequality (24) to obtain the following upper bound:

$$
\begin{array}{r}
\int_{0}^{\infty}|\mu|^{3} e^{-\mu^{2} M t}\left\{\left(\left\|r^{\prime}\right\|+|\mu| \operatorname{Lip}(\mathcal{R})+\delta^{2}\|q\|_{0,1}\right)\left|\sigma_{3}(t)-\sigma_{4}(t)\right|\right. \\
+(2 \delta\|q\|+|\mu| \operatorname{Lip}(\mathcal{R}))\|H-\bar{H}\|\} d t
\end{array}
$$


Using the definition of $\delta$ given by (34), and using the order symbol for notational convenience, the integral (43) is given by

$$
\begin{aligned}
\int_{0}^{\infty}|\mu|^{3} e^{-\mu^{2} M t}\left\{\left(\left\|r^{\prime}\right\|\right.\right. & +O(|\mu|))\left|\sigma_{3}(t)-\sigma_{4}(t)\right| \\
& \left.+|\mu|\left(\frac{2\|q\| k M}{|\lambda|}+\operatorname{Lip}(\mathcal{R})\right)\|H-\bar{H}\|\right\} d t
\end{aligned}
$$

To estimate $\left|\sigma_{3}(t)-\sigma_{4}(t)\right|$, we proceed as in the estimate for (26). Here, the required Lipschitz estimate for $\mathcal{G}$ is given by

$$
\begin{aligned}
& |\mathcal{G}(\sigma, t, H, \mu)-\mathcal{G}(\bar{\sigma}, t, \bar{H}, \mu)| \\
& \quad \leq\left(|\mu||\lambda|+2|\mu|^{2}|\nu| \delta+|\mu|^{3} \operatorname{Lip}(\mathcal{S})\right)\|H-\bar{H}\|+|\mu|^{3} \operatorname{Lip}(\mathcal{S})|\sigma-\bar{\sigma}|
\end{aligned}
$$

There are two cases. If $\operatorname{Lip}(\mathcal{S})=0$, then we obtain

$$
\left|\sigma_{3}(t)-\sigma_{4}(t)\right| \leq|\mu|(|\lambda|+O(|\mu|))\|H-\bar{H}\| t,
$$

while, for $\operatorname{Lip}(\mathcal{S}) \neq 0$, an application of Gronwall's Lemma yields

(46) $\left|\sigma_{3}(t)-\sigma_{4}(t)\right| \leq \frac{1}{|\mu|^{2}}\left(\frac{|\lambda|+O(|\mu|)}{\operatorname{Lip}(\mathcal{S})}\right)\left(e^{|\mu|^{3} \operatorname{Lip}(\mathcal{S}) t}-1\right)\|H-\bar{H}\|$.

When the Lipschitz inequality (45) or (46) is inserted into the integral (44) and the integration is performed, straightforward but lengthy simplifications of the resulting expression yield the following upper bound

$$
\begin{array}{r}
\int_{0}^{\infty}|\mu|^{3} e^{-\mu^{2} M t} \mid \mathcal{Q}\left(\sigma_{3}(t), t+\right. \\
\quad, H, \mu)-\mathcal{Q}\left(\sigma_{4}(t), t+\tau, \bar{H}, \mu\right) \mid d t \\
\leq\left(\frac{\|r\|_{0,1}|\lambda|}{M^{2}}+O(|\mu|)\right)\|H-\bar{H}\|
\end{array}
$$

To estimate the second summand of the right hand side of inequality (42), we note that, using the inequality (22) to obtain an upper bound for $\left|\mathcal{Q}\left(\sigma_{4}(t), t+\tau, \bar{H}, \mu\right)\right|$, the problem reduces to finding an estimate for the integral

$$
\begin{aligned}
\int_{0}^{\infty} \mu^{3} \mid \Phi(t+ & \tau, \tau, H)-\Phi(t+\tau, \tau, \bar{H}) \mid d t \\
& =\int_{0}^{\infty} \mu^{3}\left|e^{-\mu^{2} \int_{0}^{t} p\left(\sigma_{3}(u)\right) d u}-e^{-\mu^{2} \int_{0}^{t} p\left(\sigma_{4}(u)\right) d u}\right| d t
\end{aligned}
$$


We apply the Mean Value Theorem to the function $x \mapsto e^{x}$ to obtain the following simple fact: If $x$ and $y$ are real numbers, there is a number $c$ between $x$ and $y$, such that

$$
\left|e^{x}-e^{y}\right| \leq e^{c}|x-y|
$$

Note that both exponents appearing in the integrand on the right hand side of the equation (48) lie to the left of the number $-\mu^{2} M t$. Thus, we have

$$
\begin{aligned}
|\Phi(t+\tau, \tau, H)-\Phi(t+\tau, \tau, \bar{H})| & \leq e^{-\mu^{2} M t} \int_{0}^{t}\left|p\left(\sigma_{3}(u)\right)-p\left(\sigma_{4}(u)\right)\right| d u \\
& \leq e^{-\mu^{2} M t} \operatorname{Lip}(p) \int_{0}^{t}\left|\sigma_{3}(u)-\sigma_{4}(u)\right| d u .
\end{aligned}
$$

The Lipschitz inequalities (45) and (46) must be separately inserted into the integral in (49) and the resulting elementary integral must be evaluated. Once this is accomplished, the result must be inserted into the right hand side of (48) where again an elementary improper integral must be evaluated. After straightforward but lengthy simplifications of the resulting expression, and after taking into account the estimate for $\left|\mathcal{Q}\left(\sigma_{4}(t), t+\tau, \bar{H}, \mu\right)\right|$, we obtain the following upper bound

$$
\begin{aligned}
\int_{0}^{\infty}|\mu|^{3} \mid \mathcal{Q}\left(\sigma_{4}(t), t+\tau\right. & , H, \mu)|| \Phi(t+\tau, \tau, H)-\Phi(t+\tau, \tau, \bar{H}) \mid d t \\
\leq & \left(\frac{\operatorname{Lip}(p)\|r\|_{0,1}|\lambda|}{M^{3}}+O(|\mu|)\right)\|H-\bar{H}\|
\end{aligned}
$$

In view of the inequality (15) in the form (39) and the hypothesis given in equation (14), the sum of the leading terms (in powers of $\mu$ ) of the estimates (47) and (50) does not exceed unity. Thus, if $\mu$ is sufficiently small, the operator $\Lambda$ is a contraction.

In order to apply Theorem 2.4 , it is convenient to have a version of the result for the system (11) that is obtained when an oscillator is partially averaged at a resonance where the Melnikov function vanishes. The precise result is stated in the following corollary.

Corollary 2.6. Consider the differential equation (11) obtained by partially averaging the system (5) at a resonance $I=I_{0}$ where the perturbation terms have zero average; that is, where $\langle F\rangle$ and $\langle G\rangle$, as defined in equation (8), both vanish, and define

$$
M:=\min _{0 \leq \sigma \leq 2 \pi}\left|\left\langle F_{I}\right\rangle(\sigma)\right|>0 .
$$


If $\omega^{\prime}\left(I_{0}\right) \neq 0$,

$$
\begin{aligned}
& 5 M>\operatorname{Lip}\left(\left\langle F_{I}\right\rangle(\sigma)\right), \\
& M^{2} \geq 6\left|\omega^{\prime}\left(I_{0}\right)\right|\|h\|_{0,1},
\end{aligned}
$$

where $h$ is defined as in (9), and if $\epsilon>0$ is sufficiently small, then there is a Lipschitz continuous invariant torus for (11) near the unperturbed resonant torus corresponding to $I=I_{0}$. In particular, for $\epsilon>0$ sufficiently small, the differential equation (1) has a Lipschitz continuous invariant torus near its corresponding unperturbed resonant torus.

A very important remark about Corollary 2.6 concerns the hypothesis (52). Note that the function $h$ and the averaging transformation required to obtain the differential equation (11) both depend on the functions and on the parameters appearing in the original differential equation (1) in a complicated manner. In particular, it is very likely that, in practice, the quantity $M$ and the function $h$ both depend on some of the same parameters of the original system. Thus, in general, hypothesis (52) can not be satisfied by simply requiring $M$ to be sufficiently large. This delicate point will be addressed again in the next section.

Inequality (52) is not sharp. In fact, in view of the inequality (33), it can be replaced by

$$
M^{2} \geq(3+2 \sqrt{2})\left|\omega^{\prime}\left(I_{0}\right)\right|\|h\|_{0,1} .
$$

Also, we know from the example given by the system (13), that the number $3+2 \sqrt{2}$ can not be replaced by a number less than four. A sharp bound is not known.

The condition in Theorem 2.4 and Corollary 2.6 that $\langle F\rangle$ vanishes on the unperturbed resonant invariant torus is just the condition that the subharmonic Melnikov function vanishes on this invariant torus, a necessary condition for the torus to persist. The apparently nongeneric additional condition that $\langle G\rangle$ vanishes was imposed for convenience and also because it is known to hold for several examples. This condition does not seem to have a simple geometric interpretation. Also, it is not known if this condition is necessary for the existence of a perturbed invariant torus. Likewise, the precise form of the inequalities in the hypotheses of Theorem 2.4 are obtained from the method used in the proof. Sharp estimates are not known.

Finally, we note that we can formulate and prove a theorem similar to Theorem 2.4, that gives conditions for the existence of a Lipschitz continuous invariant torus for system (5), whose hypotheses are stated in 
terms of the corresponding system when the averaging procedure is carried only to second order. However, the hypotheses will then be stated as inequalities involving $M$ and the $(0,1)$ norm of the unknown perturbation terms of order $\epsilon^{3 / 2}$. In an application, when the system depends on parameters, such a result would not be useful because the dependence of the higher order terms on the parameters would be unknown. The advantage of averaging to third order is clear: The required norm estimates involve only the function $h$; a function that is defined in terms of averages of terms appearing in the original system.

\section{Examples}

To apply our result on the existence of invariant manifolds to a "real" oscillator, we will have to transform the given oscillator to action angle variables. Perhaps the most important models of physical interest where the action angle variables can be constructed explicitly, albeit, with the use of elliptic functions, are the pendulum and Duffing's equation. Applications of the results of this paper to physical models obtained as perturbations of these systems will be the subject of future research, cf. [3, Section 4.1] and the remarks at the end of this section. However, to give a transparent illustration of an application of our theory here, it seems appropriate to consider an example where the coordinate transformations do not require the introduction of special functions. We will analyze a slight modification of an example with this property introduced in $[\mathbf{5}]$ and $[\mathbf{6}]$; namely, the system

$$
\begin{aligned}
& \dot{x}=y\left(1-x^{2}-y^{2}\right)+\epsilon\left[\delta x-x\left(x^{2}+y^{2}\right)+\gamma x \cos (\Omega t)\right], \\
& \dot{y}=-x\left(1-x^{2}-y^{2}\right)+\epsilon\left[\delta y-y\left(x^{2}+y^{2}\right)\right] .
\end{aligned}
$$

The unperturbed system (53) is a scaled harmonic oscillator with a regular period annulus. Moreover, it can be transformed to action angle variables by a trigonometric change of coordinates. In fact, the transformation

$$
x=\sqrt{2 I} \sin \vartheta, \quad y=\sqrt{2 I} \cos \vartheta
$$

converts system (53) to the form

$$
\begin{aligned}
& \dot{I}=\epsilon\left[2 \delta I-4 I^{2}+2 \gamma I \sin ^{2} \vartheta \cos (\Omega t)\right], \\
& \dot{\vartheta}=1-2 I+\epsilon[\gamma \sin \vartheta \cos \vartheta \cos (\Omega t)] .
\end{aligned}
$$

Define

$$
T(I):=\frac{2 \pi}{1-2 I}, \quad \omega(I):=1-2 I,
$$


and note that the resonance relation is given here by

$$
\omega\left(I_{0}\right)=\frac{n}{m} \Omega .
$$

Also, note that

$$
\omega^{\prime}\left(I_{0}\right)=-2
$$

that is, the period annulus - here the entire punctured plane - is regular.

The Melnikov function, at the $(m: n)$ resonance for the system (53), is easily computed directly from definition (4). In fact, it is given by

$$
M^{m: n}(\phi)= \begin{cases}\omega\left(I_{0}\right)\left[2 \delta I_{0}-4 I_{0}^{2}-\left(2 \pi \gamma I_{0} / \Omega\right) \cos (2 \phi)\right], & \text { if } n=1, m=2, \\ \omega\left(I_{0}\right)\left(2 \delta I_{0}-4 I_{0}^{2}\right), & \text { otherwise }\end{cases}
$$

From equation $(57)$, we see that only the $(2,1)$ resonance is excited. For a discussion of the local dynamics of the system near this resonance, see reference [5] or [6]. The Melnikov function at the other resonant tori either has a fixed sign, or it vanishes identically.

This indicates, as mentioned in the introduction, that the orbits of the perturbed system drift away from the unperturbed resonant tori, whose Melnikov function has a fixed sign, in a direction determined by the sign. Moreover, for sufficiently small $\epsilon$, none of the perturbed orbits near these resonant tori are subharmonics.

The Melnikov function at an $(m: n)$ resonance with $(m: n) \neq(2: 1)$ vanishes when

$$
I_{0}=\frac{\delta}{2}
$$

If we view the system (53) with its parameters $\delta, \gamma$, and $\Omega$ fixed, and if there are relatively prime positive integers $m$ and $n$ so that $\delta=1-n / m$, then the Melnikov function will vanish when the action is adjusted so that equation (55) holds. Note that, in a physical application, where the same scenario occurs, it is perhaps not unreasonable to assume that $\delta$ is rational. It certainly will be rational if its value is obtained by physical measurement.

Under the assumption that equations (55) and (58) hold, we will apply Corollary 2.6. For this, we have

$$
\begin{aligned}
& F(I, \vartheta, t)=2 \delta I-4 I^{2}+2 \gamma I \sin ^{2} \vartheta \cos (\Omega t), \\
& G(I, \vartheta, t)=\gamma \sin \vartheta \cos \vartheta \cos (\Omega t) .
\end{aligned}
$$


At an $(m: n)$ resonance with $(m: n) \neq(2: 1)$, both $\langle F\rangle$ and $\langle G\rangle$ vanish. Also, after an easy computation, we find that

$$
\left\langle F_{I}\right\rangle(\sigma) \equiv-2 \delta
$$

In particular, we have

$$
M=2 \delta>0
$$

and

$$
\operatorname{Lip}\left(\left\langle F_{I}\right\rangle(\sigma)\right)=0
$$

To obtain the $(0,1)$ norm of the function $h$ defined in equation (9) requires a long, but straightforward, computation using the definition of $h$ and the resonance relation. We find that $h$ is given by

$h(\sigma)=\frac{2 \gamma(1-\delta) \delta^{2}}{\left(\Omega^{2}-4(1-\delta)^{2}\right)^{2}}\left(\left(\Omega^{2}-4(1-\delta)^{2}\right)^{2}-2 \gamma(1-\delta) \cos (2 \sigma)\right) \sin (2 \sigma)$,

and that an upper bound for the $(0,1)$ norm of $h$ is given by

$$
\|h\|_{0,1} \leq \frac{6 \gamma|1-\delta| \delta^{2}}{\left(\Omega^{2}-4(1-\delta)^{2}\right)^{2}}\left(\left|\left(\Omega^{2}-4(1-\delta)^{2}\right)^{2}\right|+2 \gamma|1-\delta|\right) .
$$

In view of (56), (59), and (60), the hypotheses of Corollary 2.6 will be satisfied provided the inequality (52), or, equivalently, the inequality

$$
\frac{3}{\delta^{2}}\|h\|_{0,1} \leq 1
$$

is satisfied. Thus, for fixed $\Omega$ and $\delta$, if $\gamma$, the amplitude of the periodic "force", is sufficiently small, then there is a Lipschitz continuous invariant torus.

For the case $\Omega=1$ and $\delta=1 / 4$, our theory proves that there is an invariant Lipschitz continuous torus, near the resonant unperturbed torus given by $I_{0}=\delta / 2=1 / 8$, provided that

$$
\gamma \leq \frac{5}{12}\left(\frac{1}{3} \sqrt{13}-1\right) \approx 0.084
$$

This estimate is, of course, not sharp. 
Numerical computation of the Poincaré map — the $2 \pi / \Omega$ strobe - for the system (53) with the above parameter values quickly reveals that the dominant feature of the dynamics in the $(x, y)$ plane is an attracting invariant "circle" centered at the origin with radius approximately $1 / 2$. This confirms our theoretical prediction. In fact, the resonant torus with zero Melnikov function intersects the $(x, y)$ plane in a circle with radius $\sqrt{2 I_{0}}=1 / 2$.

As an addendum to this section, we mention that for the oscillator

$$
\ddot{\theta}+\epsilon m_{2} \dot{\theta}+\sin \theta=-\epsilon m_{1}+\epsilon B \cos \left(\Omega\left(t-t_{0}\right)\right) \sin \theta
$$

that models a rotor with constant external torque attached to an elastic support, if $\epsilon$ is taken to be a perturbation parameter, then, using elliptic functions, the unperturbed system can be transformed to action-angle variables. For this example, it turns out that, for appropriate choices of the parameters, there is a resonant invariant torus on which the Melnikov function $\langle F\rangle$ vanishes identically, the function $\langle G\rangle$ vanishes identically, the function $\left\langle F_{I}\right\rangle$ is a constant function with value $-m_{2}$, and the derivative of the frequency $\omega^{\prime}$ does not vanish. Thus, several of the hypotheses required to apply Corollary 2.6 are satisfied for this important model from applied mathematics. Unfortunately, it seems to be very difficult to determine if the inequalities (51) and (52) are also satisfied. While there is strong evidence from numerical experiments for the existence of perturbed attracting invariant tori, it remains an interesting open problem to prove that these invariant tori actually exist.

\section{A. Action Angle Coordinates}

In this section we will construct the "action angle" coordinates near a periodic orbit contained in the period annulus $\mathcal{A}$ of differential equation (2). The differential equation (2), expressed in these new coordinates, that we denote by $I$ and $\vartheta$, has the form

$$
\dot{I}=0, \quad \dot{\vartheta}=\omega(I) .
$$

Interpreted geometrically, these new coordinates are related to polar coordinates in that $I$ is a radial variable and $\vartheta$ is an angular variable. In fact, $I$ is constant on each periodic solution while $\vartheta$ changes linearly on each periodic solution. In case the system (2) is Hamiltonian, the new coordinates reduce to the usual action angle coordinates on $\mathcal{A}$.

With reference to (2), define the orthogonal system

$$
\dot{u}=f^{\perp}(u), \quad u \in X
$$


where, in oriented local coordinates, $f^{\perp}(u):=J f(u)$ with

$$
J=\left(\begin{array}{rr}
0 & -1 \\
1 & 0
\end{array}\right) .
$$

We mention that $J$ rotates vectors in the plane through a positive angle of $\pi / 2$ radians. The same symbol $J$ is often used in this context with the opposite sign.

Let $\varphi_{t}$ denote the flow of the differential equation (2), and let $\psi_{t}$ denote the flow of the differential equation (64). Also, for vectors $\xi_{1}, \xi_{2}$ in $\mathbb{R}^{2}$, define $\xi_{1} \wedge \xi_{2}:=\left\langle\xi_{1}, J \xi_{2}\right\rangle$, where the brackets denote the usual inner product in $\mathbb{R}^{2}$.

A periodic orbit $\Gamma$ of (2) has an orientation determined by its time parameterization. To specify an orientation, we define $\varepsilon:=\varepsilon(f)=1$ in case, for each $\zeta \in \Gamma$, the vector $f^{\perp}(\zeta)$ is the outer normal at $\zeta$. If $f^{\perp}(\zeta)$ is the inner normal, then $\varepsilon:=-1$. Also, the orientation of the period annulus $\mathcal{A}$ is defined to be the orientation it inherits from its constituent periodic solutions.

Choose a point $\zeta \in \mathcal{A}$ and note that there is an open interval $U \subset \mathbb{R}$ containing the origin such that the image of the map $\rho \mapsto \psi_{\rho}(\zeta)$, for $\rho \in U$, is a section $\Sigma_{\zeta}$ transverse to the orbits of system (2) in $\mathcal{A}$. Define $\Upsilon: U \times \mathbb{R} \rightarrow \mathcal{A}$ by

$$
\Upsilon(\rho, \phi)=\varphi_{\phi}\left(\psi_{\rho}(\zeta)\right)
$$

Clearly, $\Upsilon$ is smooth. In fact, $\Upsilon$ is a covering map, or, in other words, a periodic coordinate system on $\mathcal{A}$. We will see below that these are "flow box" coordinates; they straighten out the flow in a neighborhood of the periodic orbit contains the point $\zeta$ as is seen in the unperturbed system (69).

To construct the action angle coordinates, we begin with the map $\Upsilon$ defined by (65). Diliberto's Theorem [1], used here with slightly different normalizations, states that if

$$
\begin{aligned}
b(t, \zeta) & :=\frac{\|f(\zeta)\|^{2}}{\left\|f\left(\varphi_{t}(\zeta)\right)\right\|^{2}} e^{\int_{0}^{t} \operatorname{div} f\left(\varphi_{s}(v)\right) d s}, \\
a(t, \zeta) & :=\int_{0}^{t}\left(2 \kappa(s, \zeta)\left\|f\left(\varphi_{s}(\zeta)\right)\right\|-\operatorname{curl} f\left(\varphi_{s}(\zeta)\right)\right) b(s, \zeta) d s,
\end{aligned}
$$

where $\kappa$ denotes the signed scalar curvature along the curve $t \mapsto \varphi_{t}(\zeta)$, $\zeta \in \mathcal{A}$, then

$$
\begin{aligned}
& D \Upsilon(\rho, \phi) \frac{\partial}{\partial \phi}=f(\Upsilon(\rho, \phi)) \\
& D \Upsilon(\rho, \phi) \frac{\partial}{\partial \rho}=b\left(\phi, \psi_{\rho}(v)\right) f^{\perp}(\Upsilon(\rho, \phi))+a\left(\phi, \psi_{\rho}(v)\right) f(\Upsilon(\rho, \phi)) .
\end{aligned}
$$


In other words, the matrix representation of the derivative $D \Upsilon(\rho, \phi)$ relative to the ordered bases $\{\partial / \partial \rho, \partial / \partial \phi\}$ and $\left\{f^{\perp}(\Upsilon(\rho, \phi)), f(\Upsilon(\rho, \phi))\right\}$ is given by

$$
D \Upsilon(\rho, \phi)=\left(\begin{array}{cc}
b\left(\phi, \psi_{\rho}(v)\right) & 0 \\
a\left(\phi, \psi_{\rho}(v)\right) & 1
\end{array}\right) .
$$

Since $b$ does not vanish for $\zeta \in \mathcal{A}$, it follows that $\Upsilon$ is a local diffeomorphism and, in fact, $\Upsilon$ is a covering map onto its image.

To express $(2)$ in $(\rho, \phi)$ coordinates, note first that there are smooth functions $(u, t) \mapsto p(u, t)$ and $(u, t) \mapsto q(u, t)$ such that

$$
g(u, t)=p(u, t) f^{\perp}(u)+q(u, t) f(u)
$$

for all $(u, t) \in \mathcal{A} \times \mathbb{R}$. Thus, to change system (2) to the new coordinates, we simply solve for

$$
j(u, t) \frac{\partial}{\partial \rho}+k(u, t) \frac{\partial}{\partial \phi}
$$

in the matrix equation

$$
\left(\begin{array}{ll}
b & 0 \\
a & 1
\end{array}\right)\left(\begin{array}{l}
j \\
k
\end{array}\right)=\left(\begin{array}{c}
\epsilon p \\
1+\epsilon q
\end{array}\right)
$$

to obtain

$$
\left(\begin{array}{l}
j \\
k
\end{array}\right)=\left(\begin{array}{c}
\epsilon \frac{1}{b} p \\
1+\epsilon\left(q-\frac{a}{b} p\right)
\end{array}\right) .
$$

It follows that (2), in the new coordinates, is given by

$$
\begin{aligned}
& \dot{\rho}=\epsilon \frac{1}{b\left(\phi, \psi_{\rho}(v)\right)} p(\Upsilon(\rho, \phi), t), \\
& \dot{\phi}=1+\epsilon\left(q(\Upsilon(\rho, \phi), t)-\frac{a\left(\phi, \psi_{\rho}(v)\right)}{b\left(\phi, \psi_{\rho}(v)\right)} p(\Upsilon(\rho, \phi), t)\right) .
\end{aligned}
$$

To compress notation, we write (68) in the form

$$
\dot{\rho}=\epsilon Q(\rho, \phi, t), \quad \dot{\phi}=1+\epsilon R(\rho, \phi, t) .
$$

Define a second change of coordinates by

$$
\rho=\beta(I), \quad \phi=\alpha(I) \vartheta
$$

where $I \mapsto \alpha(I)$ and $I \mapsto \beta(I)$ are smooth functions to be specified below. Here, since the coordinate transformation must be invertible, we 
need only assume that $\alpha(I) \beta^{\prime}(I) \neq 0$. In the $(I, \vartheta)$ coordinates, (68) has the form

$$
\begin{aligned}
\dot{I}= & \epsilon \frac{1}{\beta^{\prime}(I)} Q(\beta(I), \alpha(I) \vartheta, t), \\
(71) \dot{\vartheta}= & \frac{\dot{\phi}-\vartheta \alpha^{\prime}(I) \dot{I}}{\alpha(I)}=\frac{1}{\alpha(I)} \\
& +\epsilon\left(\frac{1}{\alpha(I)} R(\beta(I), \alpha(I) \vartheta, t)-\vartheta \frac{\alpha^{\prime}(I)}{\alpha(I) \beta^{\prime}(I)} Q(\beta(I), \alpha(I) \vartheta, t)\right) .
\end{aligned}
$$

To specify the functions $\alpha$ and $\beta$ we require two auxiliary functions - the period function and the area function. To define the period function, recall that the image of the map $\rho \mapsto \psi_{\rho}(\zeta)$, for $\rho \in U$, is a section for the unperturbed flow on the period annulus $\mathcal{A}$. The period function on $\mathcal{A}$, relative to this section, is the map $\widetilde{T}: U \rightarrow \mathbb{R}$ that assigns to each $\rho \in U$ the minimum period of the solution of (2) passing through the point $\phi_{\rho}(\zeta) \in \mathcal{A}$. In the "standard" case, $\mathcal{A}$ is an annulus whose inner boundary is a rest point. In this case, we define the area function $\zeta \mapsto A(\zeta)$; it assigns to each $\zeta \in \mathcal{A}$ the area enclosed by the unperturbed solution through $\zeta$.

The function $\beta$ is defined to be the solution of the initial value problem

$$
\frac{d \rho}{d I}=\varepsilon \frac{2 \pi}{\widetilde{T}(\rho)} \frac{1}{\left\|f\left(\psi_{\rho}(\zeta)\right)\right\|^{2}}, \quad \rho\left(I_{0}\right)=0
$$

where, in the standard case, $I_{0}=A(\zeta) /(2 \pi)$, while, in the case when $\mathcal{A}$ has a nontrivial inner boundary, we take $I_{0}=0$. The choice of initial condition for the standard case agrees with tradition. However, a different choice of initial condition simply results in a constant translation of the "action" variable; the translated coordinates remain symplectic. The function $\alpha$ is defined by

$$
\alpha(I):=-\varepsilon \frac{\widetilde{T}(\beta(I))}{2 \pi}
$$

where $\varepsilon= \pm 1$ according to the orientation of the period annulus $\mathcal{A}$.

Using the definition $T(I):=\widetilde{T}(\beta(I))$, the system (71) has the form

$$
\begin{aligned}
\dot{I}= & \varepsilon \epsilon \frac{T(I)}{2 \pi}\left\|f\left(\psi_{\rho}(\zeta)\right)\right\|^{2} Q(\beta(I), \alpha(I) \vartheta, t), \\
\dot{\vartheta}= & -\varepsilon \frac{2 \pi}{T(I)}-\varepsilon \epsilon\left(\frac{2 \pi}{T(I)} R(\beta(I), \alpha(I) \vartheta, t)\right. \\
& \left.+\vartheta \frac{T^{\prime}(I)}{2 \pi}\left\|f\left(\psi_{\rho}(\zeta)\right)\right\|^{2} Q(\beta(I), \alpha(I) \vartheta, t)\right) .
\end{aligned}
$$


From (67), we have

$$
p=\frac{1}{\|f\|^{2}}\left\langle g, f^{\perp}\right\rangle=\frac{1}{\|f\|^{2}} f \wedge g, \quad q=\frac{1}{\|f\|^{2}}\langle f, g\rangle .
$$

Thus, in view of (68), the system (74) can be rewritten in the form

$$
\begin{aligned}
\dot{I}= & \varepsilon \epsilon \frac{T(I)}{2 \pi} \mathcal{E}(I, \vartheta) f(\Upsilon(\beta(I), \alpha(I) \vartheta)) \wedge g(\Upsilon(\beta(I), \alpha(I) \vartheta), t), \\
\dot{\vartheta}= & -\varepsilon \frac{2 \pi}{T(I)}-\varepsilon \epsilon\left[\frac{2 \pi}{T(I)}\|f(\Upsilon(\beta(I), \alpha(I) \vartheta))\|^{-2}\langle f, g\rangle\right. \\
& +\left(\vartheta \frac{T^{\prime}(I)}{2 \pi}\left\|f\left(\psi_{\beta(I)}(\zeta)\right)\right\|^{2}-\frac{2 \pi}{T(I)} a\left(\alpha(I) \vartheta, \psi_{\beta(I)}(\zeta)\right)\right) \\
& \left.\left\|f\left(\phi_{\beta(I)}(\zeta)\right)\right\|^{-2} \mathcal{E}(I, \vartheta) f \wedge g\right]
\end{aligned}
$$

where

$$
\mathcal{E}(I, \vartheta):=e^{-\int_{0}^{\alpha(I) \vartheta} \operatorname{div} f(\Upsilon(\beta(I), \alpha(I) s)) d s}
$$

Again, to compress notation, we also write (75) in the compact form

$$
\dot{I}=\epsilon F(I, \vartheta, t), \quad \dot{\vartheta}=\omega(I)+\epsilon G(I, \vartheta, t)
$$

Note that both $F$ and $G$ are $2 \pi$ periodic in $\vartheta$ and $2 \pi / \Omega$ periodic in $t$.

To prove that the action angle coordinate transformation

$$
u=\Upsilon(\beta(I), \alpha(I) \vartheta)
$$

is canonical in case the unperturbed system is Hamiltonian, it suffices to show the transformation is area preserving, that is, the Jacobian of the transformation is unity. In fact, the Jacobian is

$$
\begin{array}{r}
\operatorname{det}\left[\left(\begin{array}{rr}
-f_{2}(u) & f_{1}(u) \\
f_{1}(u) & f_{2}(u)
\end{array}\right)\left(\begin{array}{cc}
b\left(\phi, \psi_{\rho}(\zeta)\right) & 0 \\
a\left(\phi, \psi_{\rho}(\zeta)\right) & 1
\end{array}\right)\right. \\
=\frac{\left.\left.\| \begin{array}{cc}
\beta^{\prime}(I) & 0 \\
\alpha^{\prime}(I) \vartheta & \alpha(I)
\end{array}\right)\right]}{\left\|f\left(\psi_{\rho}(\zeta)\right)\right\|^{2}} b\left(\phi, \psi_{\rho}(\zeta)\right) .
\end{array}
$$


But, if $f$ is a Hamiltonian vector field, then $\operatorname{div} f=0$, and

$$
b\left(\phi, \psi_{\rho}(\zeta)\right)=\frac{\left\|f\left(\psi_{\rho}(\zeta)\right)\right\|^{2}}{\|f(u)\|^{2}},
$$

as required. Moreover, in case $f$ is the Hamiltonian vector field defined by the Hamiltonian $H$, we have $f(u)=-J \operatorname{grad} H(u)$. Recall, $\rho=\beta(I)$ and define $h:=H\left(\psi_{\rho}(\zeta)\right)$. Then,

$$
\frac{d I}{d h}=\varepsilon \frac{\widetilde{T}(\rho(h))}{2 \pi} .
$$

Thus, the derivative of the action with respect to energy is the normalized energy-period function, as it should be.

\section{References}

1. C. Chicone, Bifurcation of nonlinear oscillations and frequency entrainment near resonance, SIAM J. Math. Anal. 23 (1992), 15771608.

2. C. Chicone, Lyapunov-Schmidt reduction and Melnikov integrals for bifurcation of periodic solutions in coupled oscillators, J. Differential Equations 112 (1994), 407-447.

3. C. Chicone, Periodic solutions of a system of coupled oscillators near resonance, SIAM J. Math. Anal. 26(5) (1995), 1257-1283.

4. S. P. Diliberto, On systems of ordinary differential equations, in "Contributions to the Theory of Nonlinear Oscillations," Annals of Mathematics Studies 20, Princeton University Press, Princeton, 1950.

5. B. Greenspan and P. Holmes, Repeated resonance and homoclinic bifurcation in a periodically forced family of oscillators, SIAM J. Math. Anal. 15 (1984), 69-97.

6. J. GuCKenheimer And P. Holmes, "Nonlinear oscillations, dynamical systems, and bifurcations of vector fields," Springer-Verlag, New York, 1986, second ed..

7. M. Levi, Nonchaotic behavior in the Josephson junction, Phys. Rev. A 37 (1988), 927-931.

8. V. K. Melnikov, On the Stability of the center for time periodic perturbations, Trans. Moscow Math. Soc. 12 (1963), 1-57.

9. J. A. Sanders and F. Verhulst, "Averaging Methods in Nonlinear Dynamical Systems," Springer-Verlag, New York, 1985. 
10. S. W. Wiggins, "Introduction to Applied Nonlinear Dynamical Systems and Chaos," Springer-Verlag, New York, 1990.

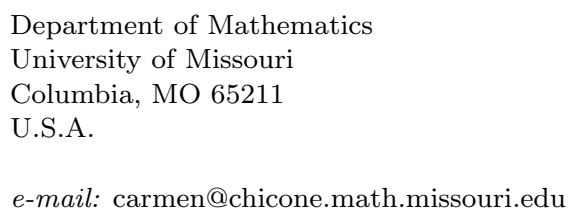

Primera versió rebuda el 30 de Novembre de 1996, darrera versió rebuda el 18 d'Abril de 1997 